\title{
Numerical simulation of temperature fields during the sublimation growth of SiC single crystals, using WIAS-HiTNIHS ${ }^{\text {is }}$
}

\author{
Jürgen Geiser ${ }^{\mathrm{a}}$, Olaf Klein ${ }^{\mathrm{b}, *}$, Peter Philip ${ }^{\mathrm{b}}$ \\ ${ }^{a}$ Humboldt-Universität zu Berlin, Department of Mathematics, Unter den Linden 6, 10099 Berlin, Germany \\ ${ }^{\mathrm{b}}$ Weierstrass Institute for Applied Analysis and Stochastics (WIAS), Mohrenstraße 39, 10117 Berlin, Germany
}

Available online 2 February 2007

\begin{abstract}
We present numerical computations of the temperature fields in axisymmetric growth apparatus for sublimation growth of silicon carbide (SiC) bulk single crystals by physical vapor transport (PVT) (modified Lely method). The results are computed using our software WIAS-HiTNIHS, the WIAS High Temperature Numerical Induction Heating Simulator; pronunciation: hit-nice, by solving the energy balance in the entire growth apparatus, taking into account the heat conduction in the solid parts as well as in gas cavities, and also accounting for the radiative heat transfer between the surfaces of the gas cavities. The insulation in a PVT growth apparatus usually consists of graphite felt, where the fibers are aligned in one particular direction, resulting in an anisotropic thermal conductivity. We show that neglecting this anisotropy can overestimate the SiC crystal's temperature by $70 \mathrm{~K}$ or underestimate the required heating power by $800 \mathrm{~W}$.
\end{abstract}

(C) 2007 Elsevier B.V. All rights reserved.

PACS: 02.60.Cb; 81.10.Bk; 44.05. +e; 47.27.Te

Keywords: A1. Anisotropic thermal conductivity; A1. Computer simulation; A1. Heat transfer; A2. Growth from vapor; A2. Single crystal growth; B2. Semiconducting silicon compounds

\section{Introduction}

Silicon carbide $(\mathrm{SiC})$ is a wide-bandgap semiconductor used as substrate material for electronic and optoelectronic applications [1]. Its chemical and thermal stability make SiC an attractive material to be used in high-temperature applications as well as in intensive-radiation environments. For an economically viable industrial use of $\mathrm{SiC}$, the availability of growth techniques for large-diameter, low-defect $\mathrm{SiC}$ boules is essential. Recent years have seen steady improvement [2] of size and quality of $\mathrm{SiC}$ single crystals grown by sublimation via physical vapor transport (PVT, also known as modified Lely method, see e.g. Refs. [3-5]). However, many problems remain, warranting further research.

\footnotetext{
This work has been supported by the DFG Research Center "MATHEON-Mathematics for key technologies" (FZT 86) in Berlin.

*Corresponding author. Tel.: + 493020372 533; fax: + 49302044975.

E-mail addresses: geiser@mathematik.hu-berlin.de (J. Geiser), klein@wias-berlin.de (O. Klein), philip@wias-berlin.de (P. Philip).
}

A PVT growth system typically consists of an inductionheated graphite crucible containing polycrystalline $\mathrm{SiC}$ source powder and a single-crystalline SiC seed (see Fig. 1). The source powder is placed in the hot zone of the growth apparatus, whereas the seed crystal is cooled by means of a blind hole, establishing a temperature difference between source and seed. In consequence, source material is transported to the seed, which grows into the reaction chamber.

The temperature distribution in the vicinity of the SiC seed and source is essential for the crystal's quality and growth rate $[4,6]$. Due to the high temperatures, experimental verification of the correlation between the design of the growth apparatus and the temperature distribution inside the growth chamber is extremely difficult and expensive. Thus, the development of numerical models and software and their application to PVT growth of $\mathrm{SiC}$ crystals has been an active field of research in recent years [6-10].

PVT growth apparatus are usually insulated by graphite felt, where the fibers are aligned in one particular direction, 


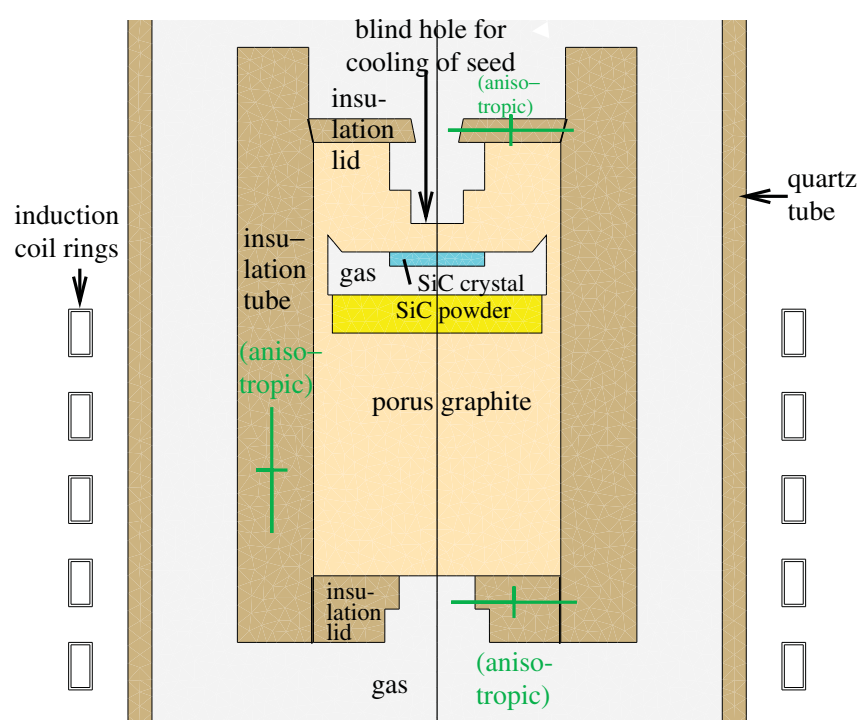

Fig. 1. Setup of growth apparatus according to Ref. [3, Fig. 2], inserted into a quartz tube.

such that the thermal conductivity of the insulation is described by an anisotropic tensor. Our results presented in Section 3 show that, for the geometry of Ref. [3], neglecting the anisotropy of the insulation's thermal conductivity can lead to an overestimation of $70 \mathrm{~K}$ in the temperature at the crystal seed's surface, or, when controlling the temperature, to an underestimation of $800 \mathrm{~W}$ in the required heating power.

\section{Modeling and numerical methods}

We employ our previously published model of heat transport in induction-heated PVT growth systems [8]. However, to allow for anisotropic thermal conductivity in the thermal insulation of the growth apparatus, instead of using the ordinary heat flux $-\kappa_{m}(T) \nabla T$ with an isotropic thermal conductivity $\kappa$, potentially depending on the absolute temperature $T$ and on the material $m$, in this paper, we use the heat flux $-K_{m}(T) \nabla T$, where $K_{m}$ is the potentially anisotropic thermal conductivity tensor. Assuming that the fiber alignment in the insulation material and the resulting anisotropy do not vary with the temperature, $K_{m}$ is a diagonal matrix of the following form:

$K_{m}(T)=\left(\kappa_{i, j}^{m}(T)\right) \quad$ where

$\kappa_{i, j}^{m}(T)= \begin{cases}\alpha_{i}^{m} \kappa_{\mathrm{iso}}^{m}(T) & \text { for } i=j, \\ 0 & \text { for } i \neq j .\end{cases}$

Here, $\kappa_{\text {iso }}^{m}(T)$ is the thermal conductivity of the isotropic case, and $\alpha_{i}^{m}$ are anisotropy coefficients.

Apart from the modified, potentially anisotropic heat flux, all interface and boundary conditions are exactly as described in Ref. [8, Section 2.4]. In particular, the temperature is assumed to be continuous throughout the apparatus, and radiative heat transfer between surfaces of cavities is modeled using the net radiation method for diffuse-gray radiation as described in Ref. [8, Section 2.5], where a band approximation model is used to account for the semi-transparency of the SiC single crystal. On the outer surface of the growth apparatus, the temperature is fixed to room temperature $T_{\text {room }}=293 \mathrm{~K}$, modeling a perfect water cooling of the quartz tube and of the aluminum lids.

Induction heating causes eddy currents in the conducting materials of the growth apparatus, resulting in the heat sources due to the Joule effect. Assuming axisymmetry of all components of the growth system as well as of all relevant physical quantities, and, furthermore, assuming sinusoidal time dependence of the imposed alternating voltage, the heat sources are computed via an axisymmetric complex-valued magnetic scalar potential that is determined as the solution of an elliptic partial differential equation (see Ref. [8, Section 2.6]). To prescribe the total heating power, we follow Ref. [11, Section II], ensuring that the total current is the same in each coil ring.

All simulations presented in this article are performed for an idealized growth apparatus, treating all solid materials as homogeneous and pure, neglecting effects such as the sintering of the $\mathrm{SiC}$ source powder, changes in the porosity of the graphite, and $\mathrm{Si}$ accumulation in the insulation. Furthermore, it is assumed that the gas phase is made up solely of argon, which is a reasonable assumption for simulations of the temperature distribution [12, Section 5].

For the numerical computations presented below, the nonlinear partial differential equations arising from the described mathematical heat transfer model are discretized using the finite volume method. The employed scheme, including the discretization of nonlocal terms stemming 
from the modeling of diffuse-gray radiation, was previously described in Ref. [13]; modifications to allow for the anisotropic thermal conductivity are treated in Ref. [14]. Also in Ref. [14], for some simple anisotropic test cases, we verified the accuracy of our finite volume scheme, comparing the numerical results with known exact solutions and determining the numerical convergence rate.

The finite volume discretization of the nonlocal radiation terms involves the calculation of visibility and view factors. The method used is described in Ref. [12, Section 4]. The discrete scheme was implemented as part of our software WIAS-HiTNIHS which is based on the program package pdelib [15]. In particular, pdelib uses the grid generator Triangle [16] to produce constrained Delaunay triangulations of the domains, and it uses the sparse matrix solver PARDISO [17,18] to solve the linear system arising from the linearization of the finite volume scheme via Newton's method.

\section{Numerical experiments}

All numerical simulations presented in the following were performed for a growth apparatus based on Ref. [3, Fig. 2], which is displayed in Fig. 1. For radius and
Experiment (1):

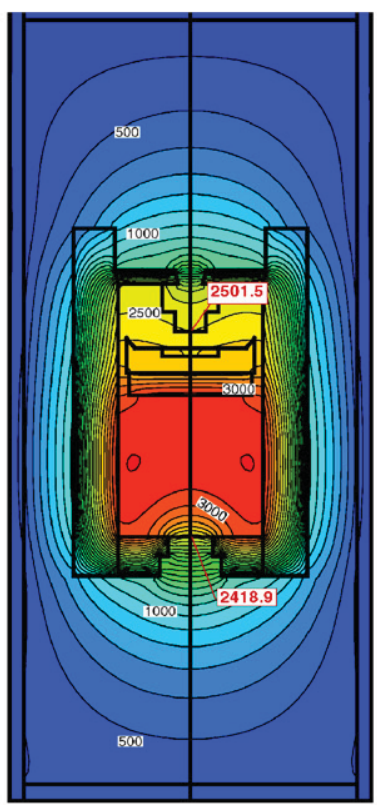

Experiment (2):

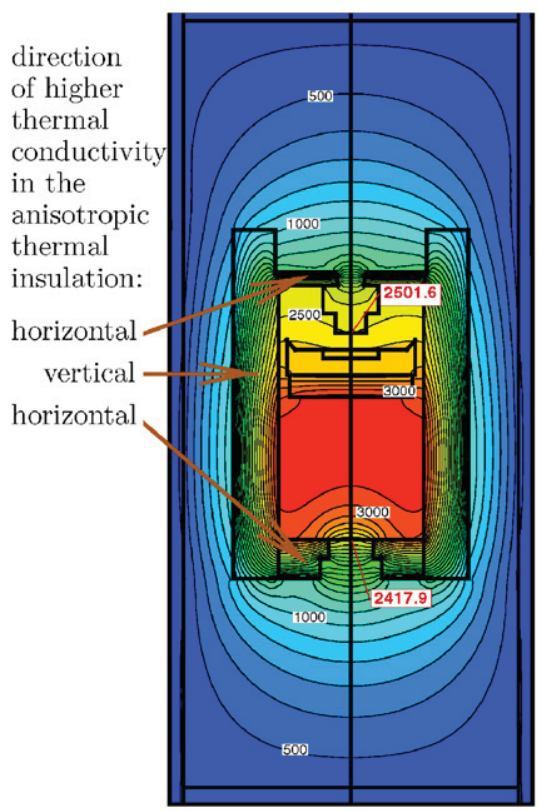

Experiment (1):

Insulation isotropic, i.e. $\left(\alpha_{r}^{6}, \alpha_{z}^{6}\right)=(1,1)$, $\left(\alpha_{r}^{7}, \alpha_{z}^{7}\right)=(1,1)$. Heating power: $10.0 \mathrm{~kW}$. Max. temp.: $3201.9 \mathrm{~K}$.

Experiment (2):

Insulation anisotropic, i.e. $\left(\alpha_{r}^{6}, \alpha_{z}^{6}\right)=(1,4)$ (tube) $\left(\alpha_{r}^{7}, \alpha_{z}^{7}\right)=(4,1)$ (lids). Heating power: $10.8 \mathrm{~kW}$. Max. temp.: $3182.4 \mathrm{~K}$.

Experiment (3):

Insulation anisotropic, i.e. $\left(\alpha_{r}^{6}, \alpha_{z}^{6}\right)=(1,4)$ (tube), $\left(\alpha_{r}^{7}, \alpha_{z}^{7}\right)=(4,1)$ (lids).

Heating power: $10.0 \mathrm{~kW}$.

Max. Temp.: $3078.3 \mathrm{~K}$.

Experiment (3):

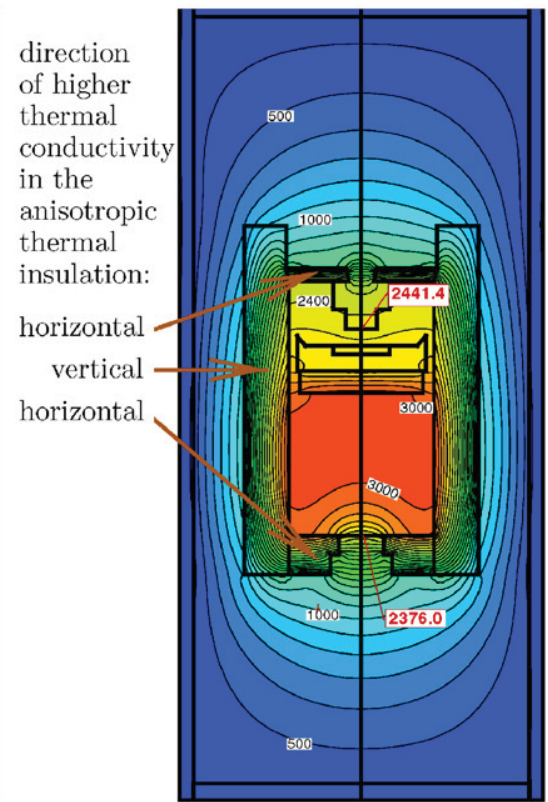

Fig. 2. Computed temperature fields with isotropic (Exp. (1)) and anisotropic (Experiments (2) and (3)) thermal insulation (cf. Fig. 3). The isotherms are spaced at $100 \mathrm{~K}$. 
height of this apparatus, we use 8.4 and $25 \mathrm{~cm}$, respectively. Moreover, the apparatus is placed inside a quartz tube measuring $13 \mathrm{~cm}$ in radius and $57.2 \mathrm{~cm}$ in height (see Fig. 1), closed with aluminum lids at the top and at the bottom.

The quartz tube lies inside five hollow, rectangularshaped copper induction rings, where the coil's lower and upper rim are at -2 and $14 \mathrm{~cm}$, respectively. For the precise geometric proportions of the coil rings see Ref. [10, Fig. 2].

As can be seen in Fig. 1, the considered apparatus consists of seven materials: graphite crucible, $\mathrm{SiC}$ crystal seed, gas enclosure, SiC powder source, quartz, insulation tube, and insulation lid. Except for the insulation materials, the material data used for the above materials during the following numerical experiments are precisely the data provided in the appendices of Refs. [10,12,19]. The material data we use for the insulation are also provided in Ref. [12, Appendix 2.2], except that, here, we use anisotropic thermal conductivities as described below.
In particular, for the isotropic parts $\kappa_{\text {iso }}^{1}(T), \kappa_{\text {iso }}^{2}(T)$, $\kappa_{\text {iso }}^{3}(T)$ of the thermal conductivity tensors (cf. Eq. (1)) of the gas enclosure, graphite crucible, and $\mathrm{SiC}$ crystal seed, we use the functions given by (A.1), (A.3b), and (A.7b) in Ref. [12], respectively; for $\kappa_{\text {iso }}^{4}(T)$ (SiC powder source), we use Ref. [19, (A.1)], and for $\kappa_{\text {iso }}^{5}(T)$ (quartz), we use Ref. [10, (A.1c)]. Moreover, for the isotropic parts $\kappa_{\text {iso }}^{6}(T)$ and $\kappa_{\text {iso }}^{7}(T)$ of the thermal conductivity tensors in the two insulation materials, i.e. the insulation tube and lids, function (A.4b) in Ref. [12] is used.

To assess the influence of the insulation's anisotropic thermal conductivity on the temperature field in the growth apparatus, we vary the anisotropy coefficients $\left(\alpha_{r}^{6}, \alpha_{z}^{6}\right)$ and $\left(\alpha_{r}^{7}, \alpha_{z}^{7}\right)$ for the insulation tube and lids, respectively, while keeping $\left(\alpha_{r}^{m}, \alpha_{z}^{m}\right)=(1,1)$ for all other materials $m \in\{1, \ldots, 5\}$. The angular frequency used for the induction heating is $\omega=2 \pi f$, where $f=10 \mathrm{kHz}$. For the prescribed average total heating power, we use $P=10.0 \mathrm{~kW}$ in our
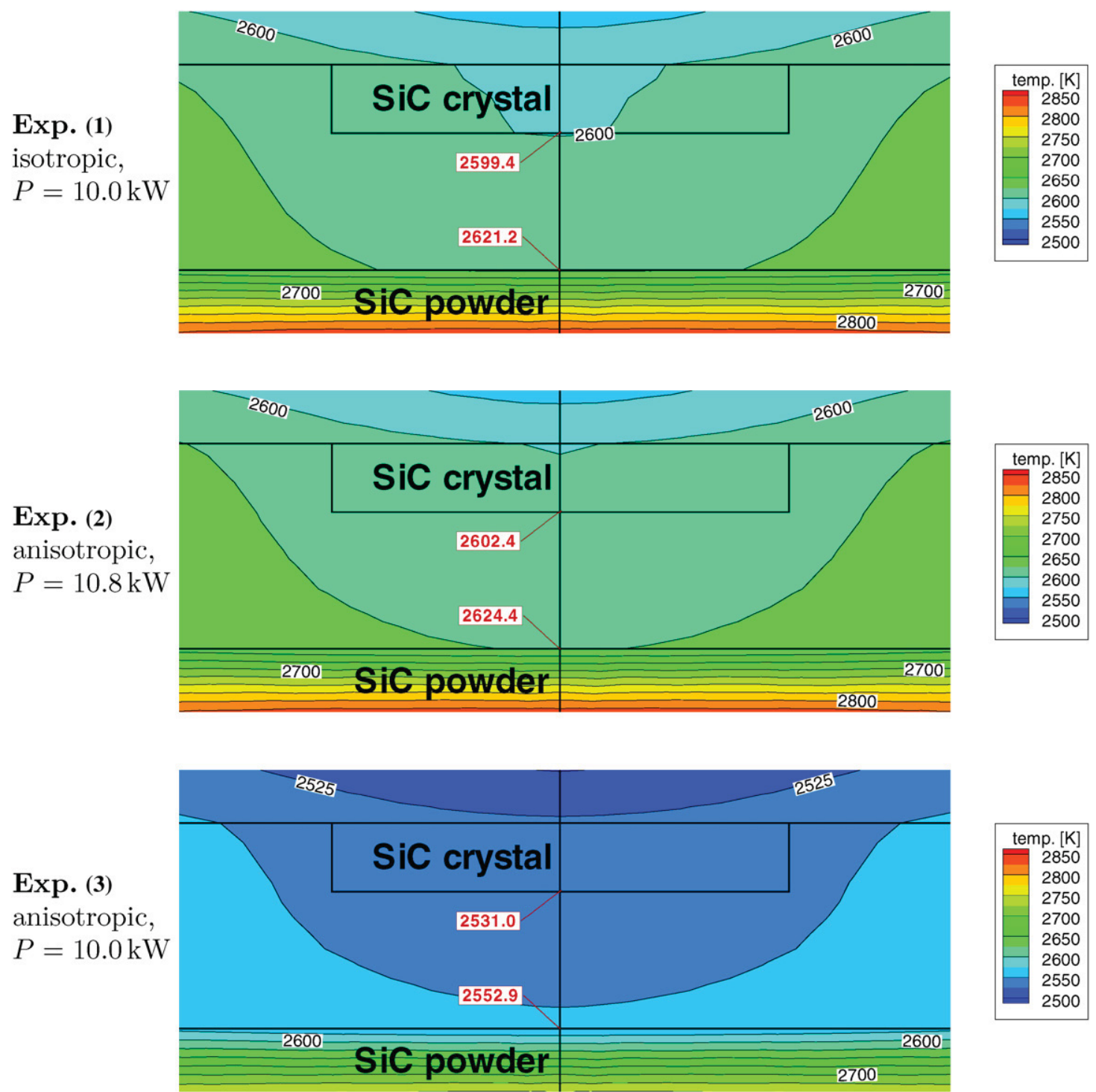

Fig. 3. Close-ups of the $\mathrm{SiC}$ crystal for the computed temperature fields with isotropic (Exp. (1)) and anisotropic (Experiments (2) and (3)) thermal insulation (cf. Fig. 2). The isotherms are spaced at $25 \mathrm{~K}$. 
first and third numerical experiments, while $P=10.8 \mathrm{~kW}$ in our second numerical experiment. We proceed to compare and discuss the results of these three numerical experiments, which we will refer to as Experiments (1)-(3). We note that our computed temperature fields (cf. Fig. 3) agree qualitatively with the experimental results in Ref. [20], where a similar apparatus was studied.

In Exp. (1), as a reference, we compute the temperature field using an isotropic thermal conductivity, i.e. $\left(\alpha_{r}^{6}, \alpha_{z}^{6}\right)$ $=\left(\alpha_{r}^{7}, \alpha_{z}^{7}\right)=(1,1)$. The result is depicted in the upper left of Fig. 2 and a close-up is shown in Fig. 3 (top).

During growth experiments performed at the Institute for Crystal Growth (IKZ) in Berlin, the anisotropy factor between the directions parallel and perpendicular to the insulation fibers was typically in the range 1-4. Given an anisotropic insulation material, one can improve the insulation's effectiveness by an appropriate apparatus design: for the insulation tube surrounding the graphite crucible, one has to ensure that the insulation is more effective radially, whereas for the lids at top and bottom, one has to ensure that the insulation is more effective vertically. This corresponds to settings $\left(\alpha_{r}^{6}, \alpha_{z}^{6}\right)=(1,4)$ in the insulation tube and $\left(\alpha_{r}^{7}, \alpha_{z}^{7}\right)=(4,1)$ in the insulation lids (cf. Fig. 1). Therefore, we use these settings in Experiments (2) and (3). Moreover, in Exp. (2), we use the bisection method to determine the heating power such that the temperature established at the upper blind hole is within $0.1 \mathrm{~K}$ of the corresponding temperature in Exp. (1) (see lefthand pictures in Fig. 2). We find that the required heating power is some $800 \mathrm{~W}$ higher than in the isotropic case. The result of Exp. (2) is shown in the lower left of Fig. 2 with the corresponding close-up in Fig. 3 (middle).

In Exp. (3), we use the same anisotropic settings for $\left(\alpha_{r}^{6}, \alpha_{z}^{6}\right)$ and $\left(\alpha_{r}^{7}, \alpha_{z}^{7}\right)$ as in Exp. (2), but we use $P=10.0 \mathrm{~kW}$ as in the isotropic Exp. (1). The result is shown in the lower right of Fig. 2 with the corresponding close-up in Fig. 3 (bottom). Fig. 3 shows that the temperature at the crystal seed's surface is overestimated by some $70 \mathrm{~K}$ in the isotropic case. Unawareness of such a temperature difference can cause the growth of unwanted SiC polytypes.

\section{Conclusions}

Based on a model describing the heat transfer in PVT growth systems, including RF heating, heat conduction allowing for anisotropic thermal conductivity tensors, and radiation between cavity surfaces, we used our simulation software WIAS-HiTNIHS to conduct numerical investigations of temperature fields in PVT growth apparatus. It was shown that neglecting the anisotropy of the thermal conductivity in the apparatus insulation, can lead to significant errors in the temperature values and in the required heating power, when calculating the temperature field in the growth chamber. In particular, the presented results show that the software tool WIAS-HiTNIHS, due to its capability to account for materials with anisotropic thermal conductivities, constitutes an improved tool for computer-aided PVT growth system optimization.

\section{Acknowledgment}

This work has been done in close cooperation with the Institute of Crystal Growth (IKZ), Berlin, Germany.

\section{References}

[1] R. Madar, J. Camassel, E. Blanquet (Eds.), Mater. Sci. Forum 457-460, (2004) (Part II), in: Proceedings of Silicon Carbide and Related Materials ICSCRM, Lyon, France, October 5-10, Trans Tech Publications Ltd, 2004 (Chapters 9-11).

[2] H. Hobgood, M. Brady, M. Calus, J. Jenny, R. Leonard, D. Malta, S. Müller, A. Powell, V. Tsvetkov, R. Glass, C. Carter Jr., Mater. Sci. Forum 457-460 (2004) 3 in: Proceedings of the 10th International Conference on Silicon Carbide and Related Materials, October 5-10, 2003, Lyon, France.

[3] M. Pons, M. Anikin, K. Chourou, J. Dedulle, R. Madar, E. Blanquet, A. Pisch, C. Bernard, P. Grosse, C. Faure, G. Basset, Y. Grange, Mater. Sci. Eng. B 61-62 (1999) 18.

[4] H.-J. Rost, D. Siche, J. Dolle, W. Eiserbeck, T. Müller, D. Schulz, G. Wagner, J. Wollweber, Mater. Sci. Eng. B 61-62 (1999) 68.

[5] H.-J. Rost, J. Doerschel, K. Irmscher, D. Schulz, D. Siche, J. Crystal Growth 257 (2003) 75.

[6] Q.-S. Chen, H. Zhang, V. Prasad, C. Balkas, N. Yushin, Trans. ASME-J. Heat Transfer 123 (6) (2001) 1098.

[7] R. Ma, H. Zhang, V. Prasad, M. Dudley, Crystal Growth Des. 2 (3) (2002) 213.

[8] O. Klein, P. Philip, J. Sprekels, Interfaces Free Boundaries 6 (2004) 295.

[9] J. Meziere, M. Pons, L.D. Cioccio, E. Blanquet, P. Ferret, J. Dedulle, F. Baillet, E. Pernot, M. Anikin, R. Madar, T. Billon, J. Phys. Condens. Matter 16 (2004) S1579.

[10] J. Geiser, O. Klein, P. Philip, Crystal Growth Des. 6 (9) (2006) 2021.

[11] O. Klein, P. Philip, IEEE Trans. Mag. 38 (3) (2002) 1519.

[12] O. Klein, P. Philip, J. Sprekels, K. Wilmański, J. Crystal Growth 222 (4) (2001) 832.

[13] O. Klein, P. Philip, Math. Mod. Methods Appl. Sci. 15 (2) (2005) 227.

[14] J. Geiser, O. Klein, P. Philip, Numerical simulation of heat transfer in materials with anisotropic thermal conductivity: a finite volume scheme to handle complex geometries, Preprint No. 1033, Weierstrass Institute for Applied Analysis and Stochastics (WIAS), Berlin, submitted. Available in pdf-format at /http://www.wias-berlin.de/ publications/preprints/1033/wias_preprints_1033.pdf 2005.

[15] J. Fuhrmann, T. Koprucki, H. Langmach, pdelib: an open modular tool box for the numerical solution of partial differential equations. Design patterns, in: W. Hackbusch, G. Wittum (Eds.), Proceedings of the 14th GAMM Seminar on Concepts of Numerical Software, Kiel, January 23-25, 1998, University of Kiel, Germany, 2001.

[16] J. Shewchuk, Triangle: Engineering a 2d quality mesh generator and delaunay triangulator, in: Proceedings of the First Workshop on Applied Computational Geometry, New York, Philadelphia, Pennsylvania, ACM, 1996, pp. 124-133.

[17] O. Schenk, K. Gärtner, W. Fichtner, BIT 40 (1) (2000) 158.

[18] O. Schenk, K. Gärtner, J. Future Gener. Comput. Syst. 20 (3) (2004) 475.

[19] O. Klein, P. Philip, J. Crystal Growth 249 (3-4) (2003) 514.

[20] M. Pons, E. Blanquet, J.M. Dedulle, I. Garcon, R. Madar, C. Bernard, J. Electrochem. Soc. 143 (11) (1996) 3727. 